\title{
Dimorfismo sexual alar em Aedes scapularis (Diptera: Culicidae)
}

\author{
Mariana Devicari ${ }^{1,2}$, Adriana Rios Lopes ${ }^{2,3}$ \& Lincoln Suesdek $k^{1,2,4}$ \\ ${ }^{1}$ Laboratório de Parasitologia, Instituto Butantan, CEP 05509-300, São Paulo, SP, Brasil, \\ http://www.butantan.gov.br/. \\ ${ }^{2}$ Instituto de Ciências Biomédicas, Pós-Graduação em Biologia da Relação Patógeno-Hospedeiro, \\ Universidade de São Paulo - USP, CEP 05508-900, São Paulo, SP, Brasil, http://www.icb.usp.br/ parasito/ \\ ${ }^{3}$ Laboratório de Bioquímica, Instituto Butantan, CEP 05509-300, São Paulo, SP, Brasil \\ ${ }^{4}$ Autor para correspondência: Lincoln Suesdek, e-mail: linrocha@butantan.gov.br
}

DEVICARI, M., LOPES, A.R. \& SUESDEK, L. Wing sexual dimorphism in Aedes scapularis (Diptera: Culicidae). Biota Neotrop. 11(2): http://www.biotaneotropica.org.br/v11n2/en/abstract?article+bn03311022011

\begin{abstract}
The sex in mosquitoes (Culicidae) is relevant for faunistic and epidemiological surveys because only females are competent to transmit pathogens. Sexual dimorphism comprising genitalia and cephalic appendages is ordinarily visible in culicids. Wings may be also dimorphic and thus be complementary to traditional sexing methods. Nevertheless, such dimorphism is not easily noticeable to direct observation. Aiming to formally describe the sexual dimorphism in Aedes scapularis, a mosquito species competent for virus and filariae, wings of males and females were compared using geometric morphometrics and multivariate statistics. We sampled populations from São Paulo and Pariquera-Açu municipalities (State of São Paulo). Concerning wing shape, the sexual dimorphism was evident from blind reclassification tests which were $100 \%$ accurate, independently of the geographical origin of samples. On the other hand, wing size was sexually dimorphic only in the population from Sao Paulo. Presumably, wing shape is evolutionarily more stable than its size, an interpretation which is in accordance with the theory posed by Dujardin (2008b). That theory indicates wing shape would be composed of genetically-determined quantitative characters, and marginally influenced by non-genetic factors, whereas wing size would be more labile and predominantly influenced by the environment.
\end{abstract}

Keywords: Aedini, Ochlerotatus, microevolution, mosquito, vector.

DEVICARI, M., LOPES, A.R. \& SUESDEK, L. Dimorfismo sexual alar em Aedes scapularis (Diptera: Culicidae). Biota Neotrop. 11(2): http://www.biotaneotropica.org.br/v11n2/pt/abstract?article+bn03311022011

Resumo: A detecção do sexo de mosquitos da família Culicidae é importante em estudos faunísticos e epidemiológicos, pois somente as fêmeas possuem competência vetora para patógenos. O dimorfismo sexual de genitália e de apêndices cefálicos é, em geral, facilmente visível em culicídeos. As asas também podem ser dimórficas e assim poderiam complementar o procedimento de sexagem. No entanto, tal distinção não é facilmente notável à observação direta. Visando descrever formalmente o dimorfismo sexual alar em Aedes scapularis, um culicídeo vetorialmente competente para arbovírus e filárias, asas de machos e fêmeas foram comparadas usando-se métodos de morfometria geométrica e análise estatística multivariada. Nestas análises, populações dos municípios São Paulo e Pariquera-Açu (Estado de São Paulo) foram amostradas. A forma das asas mostrou evidente dimorfismo sexual, o que permitiu um índice de acurácia de $100 \%$ em testes-cegos de reclassificação, independentemente da origem geográfica. Já o tamanho alar foi sexualmente dimórfico apenas na população de São Paulo. Aparentemente, a forma alar é evolutivamente mais estável que o tamanho, interpretação que está de acordo com a teoria de Dujardin (2008b), de que a forma alar de insetos seria composta por caracteres genéticos quantitativos e pouco influenciada por fatores não-genéticos, enquanto que o tamanho alar seria predominantemente determinado por plasticidade decorrente de influências ambientais.

Palavras-chave: Aedini, Ochlerotatus, microevolução, mosquito, vetor. 


\section{Introdução}

Machos e fêmeas adultos de Culicidae são em geral morfologicamente distintos, principalmente quanto às antenas, peças bucais e genitália. Existem espécies cujas asas diferem entre os sexos (Forattini 2002), porém essas características são geralmente sutis e de difícil descrição formal. Caracteres quantitativos e de difícil descrição como o formato alar, podem ser estudados por ferramentas matemáticas, como exemplificado por trabalhos que evidenciaram o dimorfismo sexual em Aedes por meio da morfometria geométrica (Jirakanjanakit et al. 2007, Henry et al. 2010). Com metodologia semelhante, há também trabalhos que abordam o desenvolvimento do formato das asas (Morales-Vargas et al. 2010) e de sua evolução (Dujardin 2008b, Jirakanjanakit et al. 2008).

Em Culicidae, considerando-se que somente as fêmeas apresentam hábito hematofágico e capacidade vetora de patógenos, torna-se primordial a distinção do sexo dos mosquitos em estudos faunísticos de caráter epidemiológico. A percepção de possíveis associações entre a forma corporal e peculiaridades eto-ecológicas de machos e fêmeas pode estimular também estudos da biologia do desenvolvimento desses insetos. Nesse contexto, a espécie Aedes (Ochlerotatus) scapularis, parece ser um bom modelo para estudo, pois além de ter importância ecológica (Forattini et al. 2000), veterinária (Macedo et al. 1998) e à saúde pública (Forattini et al. 1995), ela pode ocorrer em abundância suficiente para atender aos requisitos estatísticos de suficiência amostral.

Essa espécie é antropofílica e capaz de viver em ambientes naturais, rurais ou até urbanos (Forattini et al. 1981, 1995, Guimarães et al. 2000, Taipe-Lagos \& Natal 2003). Está amplamente distribuída no Brasil e em outros países da Região Neotropical (Forattini 2002) e possui importância para a saúde pública devido a sua competência vetora para arbovírus e filárias patogênicas (Rachou et al. 1955, Forattini et al. 1995, Macedo et al. 1998, Lima \& Ahid 2004).

Não se sabe se asas de machos e fêmeas de Ae. scapularis divergem morfologicamente como observado em outros culicídeos (Dujardin 2008b, Vidal et al. 2011). Para avaliar se há dimorfismo sexual de asas nesta espécie, machos e fêmeas provenientes de duas localidades do Estado de São Paulo foram comparados utilizando-se técnicas de morfometria geométrica sobre a venação alar.

\section{Material e Métodos}

Indivíduos adultos de Ae. scapularis foram coletados por aspiração em duas localidades no Estado de São Paulo distantes linearmente entre si $170 \mathrm{~km}$ : a) município de São Paulo (23⒉' 34" S; 46 31' 14" O), 33 fêmeas e 33 machos; b) município de

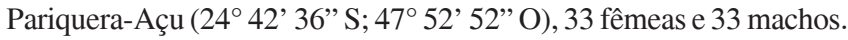
Foram utilizadas duas populações diferentes visando diversificar a amostra e eliminar possíveis peculiaridades morfológicas de uma dada população.

As asas direitas dos 132 indivíduos foram montadas entre lâmina e lamínula e fotografadas digitalmente sob estereomicroscópio Leica S6D, cuja óptica plana impede deformações periféricas de imagem. Em cada asa, as coordenadas cartesianas de 18 marcos anatômicos (Figura 1) foram digitalizadas com o auxílio do software TpsDig (Rohlf 2005).

Possíveis erros humanos na digitalização dos marcos anatômicos foram estimados comparando-se duas séries de digitalização. Tal estimativa foi feita com base no índice "repetibilidade" (Arnqvist \& Märtensson 1998) com o auxílio do software Statistica 6.0 (StatSoft). Todas as imagens de asas tiveram seus marcos anatômicos digitalizados duas vezes por cada autor e as respectivas repetibilidades foram computadas. Para cada marco anatômico, somente as coordenadas com o maior índice de repetibilidade foram usadas na análise subseqüente. Apenas os marcos anatômicos cuja repetibilidade média foi acima de $90 \%$ foram utilizados para prosseguimento da análise.

As coordenadas dos eixos Xe Y referentes aos marcos anatômicos foram submetidas à sobreposição generalizada por mínimos quadrados (Rohlf 1999). A partir das coordenadas residuais, variáveis de forma (partial warps) foram produzidas e seus componentes principais (relative warps) foram comparados entre amostras de machos e fêmeas com o auxílio do software BAC (Dujardin 2008a).

Distâncias de Mahalanobis nos eixos discriminantes foram computadas para avaliar o grau de similaridade entre as amostras. Baseados nas distâncias de Mahalanobis, testes "cegos" de reclassificação foram feitos para avaliar o poder discriminante dos caracteres alares analisados (Dujardin 2009). Para maior confiabilidade, tais testes foram efetuados excluindo-se a asa a ser classificada e reinserindo-a como dado suplementar logo após classificação do restante do grupo, conforme empregado em Dujardin (2009). Configurações de consenso do formato alar com tamanhos alares equalizados foram obtidas também pelo método de método de sobreposição pelos mínimos quadrados com o auxílio do software TpsRelW (Rohlf 2003) e permitiram a comparação da forma alar entre os sexos, sem interferência de variações isométricas de tamanho das asas.

O tamanho isométrico das asas foi determinado por cômputo de tamanho do centróide, calculado com base nas coordenadas cartesianas dos 18 marcos anatômicos (Dujardin 2008b). Valores individuais do vetor tamanho do centróide, após avaliação da normalidade e homogeneidade das variâncias pelos testes de Kolmogorov-Smirnov e Levene, foram estatisticamente comparados por meio do teste não-paramétrico Mann-Whitney. Para cada população, machos foram comparados com fêmeas. Foram feitas também comparações entre as populações de São Paulo e Pariquera-Açu independentemente

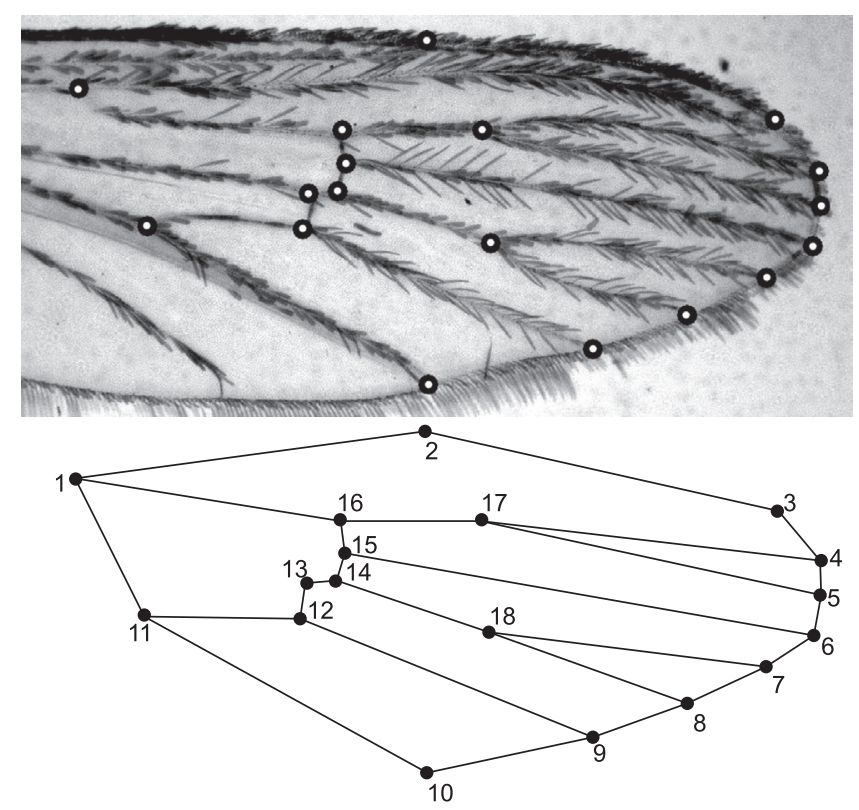

Figura.1. Acima, asa de Aedes scapularis e os 18 marcos anatômicos utilizados. Abaixo, diagrama ligando alguns marcos para facilitar a visualização da forma nas comparações alares.

Figure 1. Above, wing of Aedes scapularis and the 18 landmarks chosen. Below, diagram of links among some landmarks used to enhance visibility of wing shape in comparative analyses. 
para cada sexo para estimar a existência de variações geográficas. Nomenclatura biológica é usada aqui de acordo com Reinert (1975).

\section{Resultados}

A análise de componentes principais revelou machos e fêmeas distribuídos isoladamente no morfo-espaço dos componentes principais, permitindo a distinção inequívoca de ambos os sexos ao longo do eixo do componente principal 1 (Figura 2). Indivíduos do mesmo sexo agruparam-se independentemente da procedência geográfica.

As distâncias de Mahalanobis entre os sexos foram de 6,69 (Pariquera-Açu) e 7,30 (São Paulo). Por outro lado, comparando-se as localidades (isoladamente para cada sexo), as distâncias de Mahalanobis foram de 1,44 para fêmeas e 1,77 para machos. Os testes de reclassificação permitiram a identificação do sexo de todos os indivíduos com $100 \%$ de acerto. Observando-se as configurações de consenso do formato das asas geradas após sobreposição de Procrustes percebe-se que machos e fêmeas diferiram em todos os marcos anatômicos, sendo que a maior divergência residiu nos marcos 10 a 11 e 14 a 18 (Figura 3).

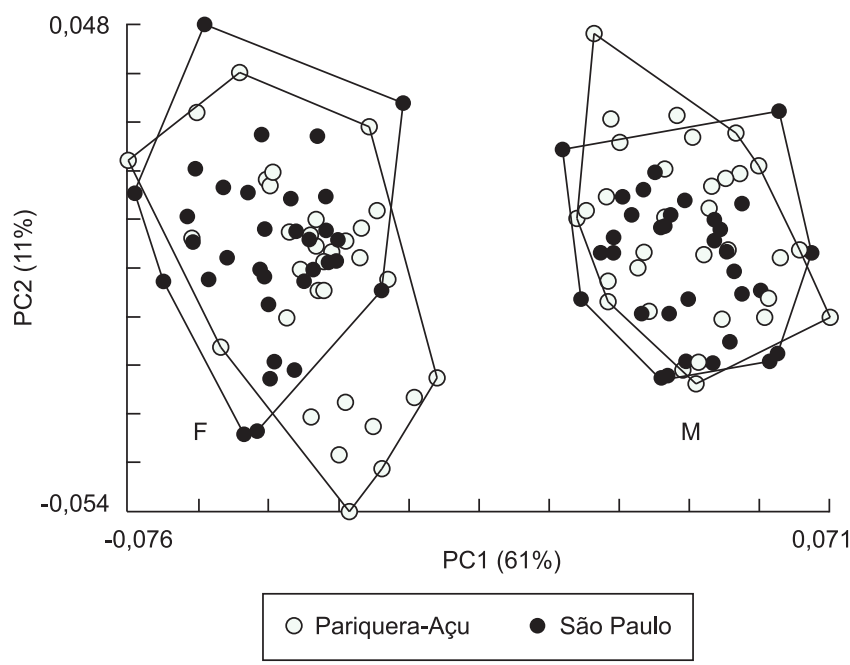

Figura.2. Morfo-espaço dos componentes principais gerados a partir da comparação multivariada das variáveis de forma alar. Indivíduos machos (M) e fêmeas (F) provenientes de Pariquera-Açu e São Paulo.

Figure 2. Morphospace of principal components yielded by multivariate comparison of wing shape variables. Males (M) and females (F) from Pariquera-Açu and São Paulo.

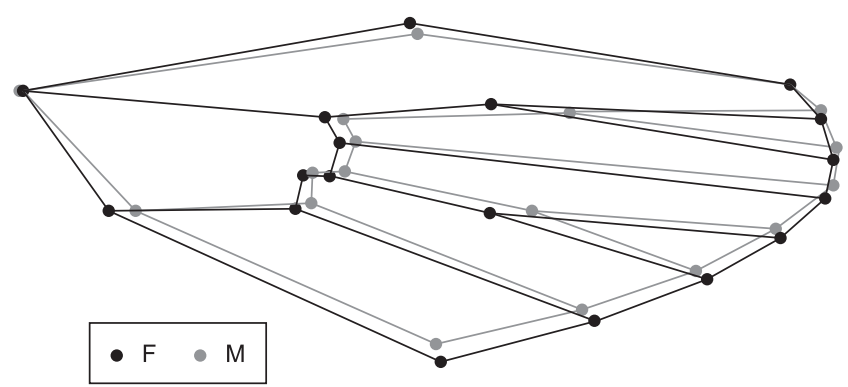

Figura 3. Diagrama das configurações-consenso da forma alar de machos e fêmeas (ambas as populações incluídas). As diferenças isométricas de tamanho foram removidas. As configurações foram sobrepostas no marco anatômico No. 1.

Figure 3. Diagram of consensus configurations of wing shape of males and females (both populations included). Isometric size divergencies were removed. Configurations were superimposed on landmark \#1.
Quanto ao tamanho das asas, as quatro amostras apresentaram distribuição Gaussiana segundo o teste de normalidade Kolmogorov-Smirnov. No entanto, as variâncias mostraram-se heterogêneas segundo o teste de Levene, e por isso as amostras foram comparadas por meio de teste não-paramétrico Mann-Whitney. O dimorfismo sexual de tamanho alar foi significativo somente na população de São Paulo, onde as fêmeas foram maiores (Mann-Whitney; $Z=-6,29 ; P<0,0001$ ). A diferença geográfica foi significativa somente entre os machos, os quais foram maiores em Pariquera-Açu (Mann-Whitney; $Z=6,23 ; \mathrm{P}<0,0001$ ). A comparação gráfica dos tamanhos médios encontra-se na Figura 4.

\section{Discussão}

O dimorfismo sexual de forma alar mostrou-se conspícuo em Ae. scapularis e a distinção entre machos e fêmeas foi inequívoca nos testes de sexagem feitos a partir de caracteres alares. Analisando-se as distâncias de Mahalanobis, a diferenciação entre os sexos teve magnitude pelo menos 3,7 vezes superior à diferenciação interpopulacional (calculada isoladamente para cada sexo), revelando que quanto à forma alar, o dimorfismo sexual foi maior que a variação geográfica.

Quanto ao tamanho alar, observou-se dimorfismo sexual somente na amostra populacional de São Paulo. Ademais, fêmeas das duas localidades diferiram quanto ao tamanho, enquanto que os machos foram semelhantes neste quesito em ambos os locais.

Os presentes dados indicam que a expressão fenotípica da forma alar é específica para machos e para fêmeas em Ae. scapularis, embora o mesmo não aconteça para tamanho. Aparentemente, a forma alar é evolutivamente mais estável que o tamanho e por essa razão pode ser utilizada com maior confiabilidade na sexagem de indivíduos desta espécie.

Tais observações e interpretações estão de acordo com a atual teoria sobre as bases biológicas do desenvolvimento alar em Culicidae (Jirakanjanakit et al. 2007, 2008, Dujardin 2008b, Morales-Vargas et al. 2010). Segundo aqueles autores, a forma alar em Culicidae é determinada por loci genéticos quantitativos, sendo assim, é herdável e minimamente influenciada por fatores epigenéticos. Já o tamanho alar, embora potencialmente herdável,

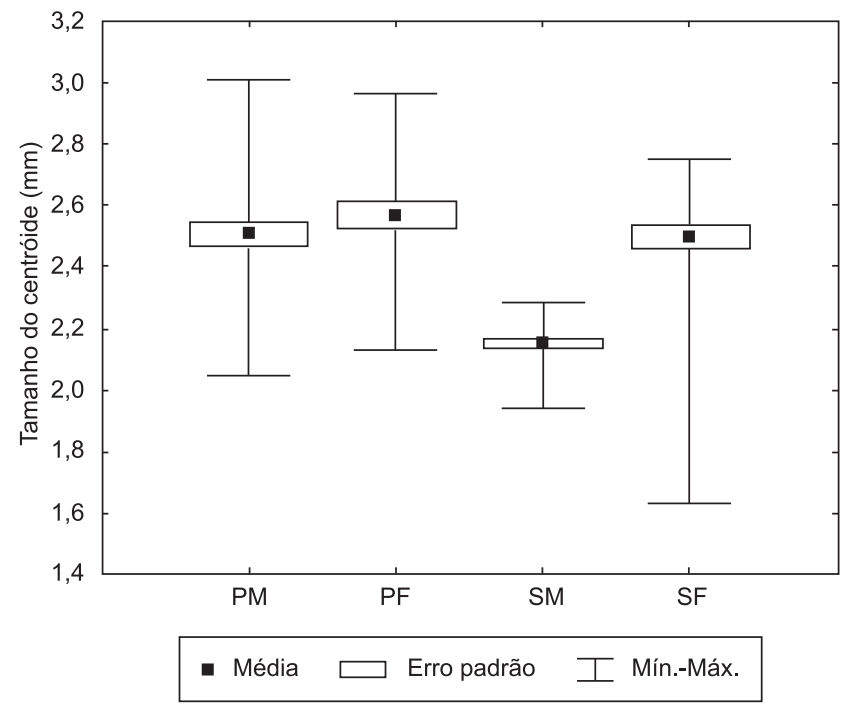

Figura 4. Comparação gráfica dos tamanhos de centróide das amostras: machos (M) e fêmeas (F) provenientes de Pariquera-Açu (P) e São Paulo (S).

Figure 4. Graphical comparison of centroid sizes of samples: males $(\mathrm{M})$ and females (F) from Pariquera-Açu (P) and São Paulo (S). 
pode ser significativamente influenciado por condições ambientais como quantidade de alimento (Jirakanjanakit et al. 2007), umidade relativa do ar (Morales-Vargas et al. 2010) e temperatura (Scott et al. 2000, Tsuda \& Takagi 2001).

O dimorfismo sexual alar deverá ser levado em consideração em alguns estudos que envolvam Ae. scapularis. De acordo com Arnell (1976) e Forattini (2002), o táxon Ae. scapularis apresenta grande variabilidade morfológica individual e possivelmente congrega um complexo de espécies ainda não caracterizadas, podendo assim ser objeto de estudos populacionais futuros.

O dimorfismo sexual em insetos e seu significado ecológico e evolutivo têm sido investigados há décadas. Como exemplos pode-se citar a correlação entre dimorfismo sexual e diferenciação ecológica entre sexos em Diptera (Atchley 1971) e a relação entre determinantes genéticos da forma alar e a fecundidade de machos e fêmeas de Orthoptera (Roff et al. 1999). Roff \& Fairbairn (1991) ressaltam que a evolução do dimorfismo sexual de asas em insetos é informativa para entendimento da evolução da capacidade dispersional de machos e fêmeas. Biomecanicamente, sabe-se que o padrão de venação alar determina as possíveis deformações aerodinâmicas das asas durante o vôo (Dudley 2000), o que reafirma a importância da geometria da asa na capacidade de vôo e dispersão de insetos.

Entre os Lepidópteros há casos de sexos dimórficos quanto a forma, coloração pigmentar e estrutural das asas (Oliver et al. 2009, Shevtsova et al. 2011). A produção de sons de corte sexual também pode ser dependente da geometria alar sexo-específica, como evidenciado em Orthoptera (Klingenberg et al. 2010).

Em suma, o senso-comum admite que caracteres diferenciais entre machos e fêmeas frequentemente representam um probelma biológico merecedor de investigação pois podem indicar ocorrência de seleção sexual (Bonduriansky 2006).

Não são conhecidas as causas do dimorfismo sexual em Ae. scapularis, nem suas implicações ecológico-evolutivas. Estaria esse dimorfismo evolutivamente influenciado por seleção sexual, já que o som produzido pelo batimento das asas de culicídeos é sexoespecífico e tem papel no reconhecimento individual precedente à cópula (Cator et al. 2009)? Para elucidar-se esta complexa questão, provavelmente será necessário aprofundar os estudos sobre a relação forma-função das asas e sobre os padrões de herança genética da forma alar.

\section{Agradecimentos}

À Cristina Chagas e Fernanda Silva Almeida pelo apoio técnico. Aos colegas Enéas de Carvalho, Fábio de Almeida e Paloma O. Vidal por suas sugestões. Ao Departamento de Epidemiologia da Faculdade de Saúde Pública da Universidade de São Paulo por ceder acesso ao posto avançado de Entomologia Médica do município de Pariquera-Açu. À FAPESP pelo auxílio regular à pesquisa \#06/02622-5.

\section{Referências Bibliográficas}

ARNELL, J.H. 1976. Mosquito studies (Diptera, Culicidae). XXXIII. A revision of the scapularis group of Aedes (Ochlerotatus). Contrib. Amer. Entomol. Inst. 13(3):1-144.

ARNQVIST, G. \& MÄRTENSSON, T. 1998. Measurement error in geometric morphometrics:empirical strategies to assess and reduce its impact on measure of shape. Acta Zool. Acad. Scient. Hungaricae. 44 (1-2):73-96.

ATCHLEY, W.R. 1971. Components of sexual dimorphism in Chironomus larvae (Diptera: Chironomidae). Am. Natur. 105 (945):455-466. http://dx.doi.org/10.1086/282737
BONDURIANSKY, R. 2006. Convergent evolution of sexual shape dimorphism in Diptera. J. Morphol. 267 (5):602-611. PMid:16477603. http://dx.doi.org/10.1002/jmor.10426

CATOR, L.J., ARTHUR, B.J, HARRINGTON, L.C. \& HOY, R.R. 2009. Harmonic convergence in the love songs of the Dengue vector mosquito. Science. 323 (5917):1077-1079. PMid:19131593. PMCid:2847473. http://dx.doi.org/10.1126/science.1166541

DUDLEY, R. 2000. The Biomechanics of Insect Flight: Form, Function, Evolution. Princeton University Press, New Jersey, 476p.

DUJARDIN, J.P. 2008a. BAC version 35 [Computer program]. Institute de recherche pour le développement, Montpellier, France. http://www.mpl. ird.fr/morphometrics/

DUJARDIN, J.P. 2008b. Morphometrics applied to medical entomology. Inf. Genet. Evol. 8:875-890. PMid:18832048. http://dx.doi.org/10.1016/j. meegid.2008.07.011

DUJARDIN, J.P. 2009. PAD version 90 [Computer program]. Institute de recherche pour le développement, Montpellier, France. http://www.mpl. ird.fr/morphometrics/.

FORATTINI, O.P. 2002. Culicidologia Médica. EDUSP, São Paulo, 864p.

FORATTINI, O.P., GOMES, A.C., SANTOS, J.L.F., GALATI, E.A.B., RABELLO, E.X. \& NATAL, D. 1981. Observações sobre atividade de mosquitos Culicidae em mata residual no Vale do Ribeira, S.Paulo, Brasil. Rev. Saúde Pública. 15:557-86. http://dx.doi.org/10.1590/S003489101981000600001

FORATTINI, O.P., KAKITANI, I., DOS SANTOS, R.L., KOBAYASHI, K.M., UENO, H.M. \& FERNANDEZ, Z. 2000. Adults Aedes albopictus and Ae. scapularis behavior (Diptera: Culidae) in southeastern Brazil. Rev. Saúde Publica. 34(5):461-7.

FORATTINI, O.P., KAKITANI, I., MASSAD, E. \& MARUCCI, D. 1995. Studies on mosquitoes (Diptera: Culicidae) and anthropic environment. 9 - Synanthropy and epidemiological role of Aedes scapularis in South-Eastern Brazil. Rev. Saúde Pública. 29:199-207. http://dx.doi. org/10.1590/S0034-89101995000300007

GUIMARÃES, A.E., GENTILE, C., LOPES, C.M. \& MELLO, R.P. 2000. Ecology of mosquitoes (Diptera: Culicidae) in areas of Serra do Mar State Park, State of São Paulo, Brazil. II - habitat distribution. Mem Inst Oswaldo Cruz. 95(1):17-28. http://dx.doi.org/10.1590/S007402762000000100002

HENRY, A., THONGSRIPONG, P., FONSECA-GONZALEZ, I., JARAMILLO-OCAMPO, N. \& DUJARDIN, J.P. 2010. Wing shape of dengue vectors from around the world. Infect. Genet. Evol. 10(2):207-214. http://dx.doi.org/10.1016/j.meegid.2009.12.001

JIRAKANJANAKIT, N., LEEMINGSAWAT, S. \& DUJARDIN, J.P. 2008. The geometry of the wing of Aedes (Stegomyia) aegypti in isofemale lines through successive generations. Inf. Genet. Evol. 8:414-421. http://dx.doi.org/10.1016/j.meegid.2007.05.004

JIRAKANJANAKIT, N., LEEMINGSAWAT, S., THONGRUNGKIAT, S., APIWATHNASORN, C., SINGHANIYOM, S., BELLEC, C. \& DUJARDIN, J.P. 2007. Influence of larval density or food variation on the geometry of the wing of Aedes (Stegomyia) aegypti. Trop. Med. \& Intl. Health. 12(11):1354-1360. http://dx.doi.org/10.1111/j.13653156.2007.01919.x

KLINGENBERG, C.P., DEBAT, V.\& ROFF, D.A. 2010. Quantitative genetics of shape in cricket wings: developmental integration in a functional structure. Evolution. 64(10):2935-2951.

LIMA, P.M. \& AHID, S.M.M. 2004. Vetor natural da Dirofilaria immitis (Nematoda) em Mossoró-RN. Caatinga. 17(2):109-114.

MACEDO, F.C., LABARTHE, N. \& LOURENÇO-DE-OLIVEIRA, R. 1998. Susceptibility of Aedes scapularis (Rondani, 1848) to Dirofilaria immitis (Leidy, 1856), an emerging zoonosis. Mem Inst Oswaldo Cruz. 4:435-37. http://dx.doi.org/10.1590/S0074-02761998000400003

MORALES-VARGAS, ER., YA-UMPHAN, P., PHUMALA-MORALES, N., KOMALAMISRA, N. \& DUJARDIN, J.P. 2010. Climate associated size and shape changes in Aedes aegypti (Diptera: Culicidae) populations from Thailand. Infect. Genet. Evol. 10:580-585. http://dx.doi.org/10.1016/j. meegid.2010.01.004 
OLIVER, J.C., ROBERTSON, K.A. \& MONTEIRO, A. 2009. Accommodating natural and sexual selection in butterfly wing pattern evolution. Proc. Royal. Soc. B-Biol. Sci. 276(1666):2369-2375.

RACHOU, R.G., LIMA, M.M., NETO, J.A.F. \& MARTINS, C.M. 1955. Inquérito epidemiológico de filariose bancroftiana em uma localidade de Santa Catarina, como fase preliminar de uma prova profilática. Constatação de transmissão extradomiciliária por um novo vetor, Aedes scapularis. Rev. Bras. Malariol. Doenças Trop. 7:51-70.

REINERT, J.F. 1975. Mosquitoes generic and subgeneric abbreviations (Diptera: Culicidae). Mosquito System. 7:105-110.

ROFF, D.A. \& FAIRBAIRN, D.J. 1991.Wing dimorphisms and the evolution of migratory polymorphisms among the Insecta. Am. Zool. 31(1):243-251.

ROFF, D.A., TUCKER, J., STIRLING, G. \& FAIRBAIRN, D.J. 1999. The evolution of threshold traits: effects of selection on fecundity and correlated response in wing dimorphism in the sand cricket. J. Evol. Biol. 12(3):535-546. http://dx.doi.org/10.1046/j.1420-9101.1999.00051.x

ROHLF, F.J. 1999. Shape statistics: Procrustes superimpositions and tangent spaces. J. Classif. 16:197-223. http://dx.doi.org/10.1007/s003579900054

ROHLF. F.J, 2003. TpsRelw, relative warps analysis. Version 1.36 [Computer program]. Department of Ecology and Evolution, State University of New York, Stony Brook, New York. http://life.bio.sunysb.edu/morph/
ROHLF, F.J. 2005. TpsDig, digitize landmarks and outlines. Version 2.05 [Computer program]. Department of Ecology and Evolution, State University of New York, Stony Brook, New York. http://life.bio.sunysb. edu/morph/

SCOTT, T.W., AMERASINGHE, P.H., MORRISON, A.C., LORENZ, L.H., Clark, G.G., STRICKMAN, D., KITTAYAPONG,P. \& EDMAN, J.D., 2000. Longitudinal studies of Aedes aegypti in Thailand and Puerto Rico: blood-feeding frequency. J. Med. Entomol. 37:89-101. http://dx.doi. org/10.1603/0022-2585-37.1.89

SHEVTSOVA, E., HANSSON, C., JANZEN, D.H. \& KJAERANDSEN, J. 2011. Stable structural color patterns displayed on transparent insect wings. Proc. Natl. Acad. Sci. 108(2):668-673. PMid:21199954. PMCid:3021046. http://dx.doi.org/10.1073/pnas.1017393108

TAIPE-LAGOS, C.B. \& NATAL, D. 2003. Abundância de culicídeos em área metropolitana preservada e suas implicações epidemiológicas. Rev. Saúde Pública. 37(3):275-9.

TSUDA, Y. \& TAKAGI, M. 2001. Survival and development of Aedes aegypti and Aedes albopictus (Diptera:Culicidae) larvae under a seasonally changing environment in Nagasaki, Japan. Environ. Entomol. 30:855-860. http://dx.doi.org/10.1603/0046-225X-30.5.855

VIDAL, P.O., ROCHA, L.S. \& PERUZIN, M.C.J. 2011. Wing diagnostic characters for mosquitoes Culex quinquefasciatus and Culex nigripalpus (Diptera; Culicidae). Rev. Bras. Entomol. 55(1) no prelo.

Recebido em 10/03/2010 Versão reformulada recebida em 19/04/2011 Publicado em 13/06/2011 